\title{
DEEP LEARNING APPROACH FOR INDIAN CURRENCY CLASSIFICATION
}

\author{
Suyash Mahesh Bahrani \\ Student, Department of Information Technology \\ Thadomal Shahani Engineering CollegeMumbai, Maharashtra, India
}

\begin{abstract}
Currency is an unavoidable part of our day-today life. Despite the rapidly expanding utilization of master cards and additional electronic payment categories, money is considerably utilized for everyday exchanges because of its comfort. The current day monetary self-service gives birth to currency recognition, which plays a vital role in the automated banking procedure. Therefore we propose a novel method for currency recognition that identifies Indian currency in different views on the scale. It is straightforward for a typical human being to comprehend and recognize any banknote easily, but it is undoubtedly troublesome for anyone with a visually impaired or blind individual to accomplish a similar task. Banknotes commonly have unique designs according to the denomination and can be sorted with surplus human errors in the bank. These errors lead to difficulties in evaluating and recognition. If computers or mobile apps recognize currency, it will immensely boost the precision of recognition and ameliorate people's workload efficiently. As money has a significant role in daily life for any business transactions, real-time detection and recognition of banknotes become necessary for a person, especially blind or visually impaired, or for a system that sorts the data.
\end{abstract}

The model which we worked on essentially classifies the currency note into distinct denominations like Rs10, Rs50, Rs100, Rs500, Rs2000. The currency will be recognized and classified by using image processing techniques, deep learning techniques. We have implemented transfer learning theory, a deep learning domain where we will reuse the weights in one or more layers from an already trained model into a new model by either maintaining the weights fixed, fine-tuning them, or adapting the weights completely when training the model. We aim to enhance techniques that have been missing in most contemporary works that have been done so far. Therefore our proposed currency recognition system can be efficiently run on the web application/mobile app, where a user would upload an image of the currency note, and it will deliver an audio output and a text output.

Keywords - Image processing, transfer learning, deep learning

\section{INTRODUCTION}

The proficiency in recognizing currency (both coins and bills) without human input is unfortunate for several applications. Currency (which encompasses paper notes and standard coins) is practically a medium for switching goods and services. It is absolutely a simple task for a human to identify denomination currency or classify the currency accordingly because our brain is qualified enough to do that. Hence most of the paper currency recognition methods implicate image processing and some classifiers, mainly neural networks. Moreover, striding towards the overall economy shall formulate the task of paper currency recognition even further demanding as the overt monetary depiction might be arduous. Hence we must glance for some inherent characterization for currency recognition. Currency has intrinsic as well as extrinsic properties. Extrinsic features are comprised of physical properties of the currency (size and width)

Nevertheless, these physical features are not credible. Occasionally currencies may get damaged during circulation. Hence the system errs to recognize damaged currencies. However, in computer vision, classifying the currency might not be that manageable primarily when there are miscellaneous cases of currencies physical conditions involving damage, crumpled notes, and even have non-uniform radiance due to wear and tear. In this thesis, we specifically use deep learning as the framework, employing convolutional neural networks(CNN) to extract paper currency features to accurately recognize the denomination of the currency covering both sides front and back. This system is used to detect the currency through the automated system through convolution neural networks. Deep learning excels in recognizing and classifying images over substantial data sets, which is also chiefly used in object category recognition. The proposed model is distinguished from widely used neural network-based models like VGG16, VGG19, Xception, InceptionV3, AlexNet, and ResNet50 in terms of training and testing accuracy. In this model, the following points are proposed: (1) data collection and processing, (2) data augmentation, (3) currency feature extraction, (4) currency identification, (5) result analysis of currency recognition. 


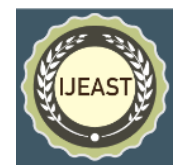

\section{REVIEW OF LITERATURE}

The classification of Indian currency notes has been addressed in several approaches. Over the years, a substantial number of researches have been executed in this field of currency note recognition. The authors have accomplished recognition based on color, texture, security features. The generally utilized methods incorporate artificial neural networks, currency characteristic comparison, principal component analysis, local feature descriptors comparison, hidden Markov models, and Naive Bayes classifier. A paper currency recognition system is developed to compile noticeable or hidden currency features for classification. Lately, various techniques were engaged in recognizing paper currencies. Hassan pour et al.(2007) introduced a reasonable method to extract visible features from the currency. However, such a procedure ceases to function when dealing with analogous sized currencies of different countries or currencies whose visible features are damaged. Prevailing research in the area of currency recognition is established on the image processing and neural networks. Vila et al.(2006) designed a currency recognition system using a symmetric mask for the paper currency signs. In this process sum of all non-masked pixel values are computed and provided as input to the neural network. Zangh et al.(2006) proposed detection based on pattern edges of the currency. In this method, the paper currency is vertically divided into several parts, and the system will discover the number of pixels denoting each edge. The pixel value achieved is later provided to the backpropagation neural network. There are many methods inferred to detect faces using neural networks. For instance, in 1994, Vaillant et al. searched for regions that may contain a face in the input image using a neural network and used another neural network to deduce if it indeed contained a face. Also, in 1998 Roley et al. proposed to detect faces using a neural network learned using the Bootstrap method, achieving high performance in detecting frontal faces. This method was later expanded so that it is robust to the rotation of the face. In 2002 Garcia et al. presented a neural network that can detect faces in input images of different sizes, various lighting conditions, and varying faces' orientation.

Further, in 2005 Osadchy et al. achieved real-time face detection and pose estimation using a Convolution Neural Network (CNN). Not only have neural networks shown their outstanding performance in face detection, but they have exemplified usefulness in detecting objects other than faces. Moreover, in recent years, training deep neural networks have become feasible, steering to detect multiple concurrent objects. Thus, we conclude that adopting transfer learning techniques, which is a deep learning domain, helps to train a pre-trained model and recognize images for adequate accuracy.

\section{METHODOLOGY}

The proposed explanation is reasonably straightforwardclassifying Indian currency notes using deep learning approaches. There are various image classifier models, each following its methodology. The dataset used is images of currency notes segregated into training and testing set by the ratio of $85: 15$, as shown below. There are approximately 2570 images of different denominations of Indian currency.

\begin{tabular}{|l|l|l|l|}
\hline Notes & Training & Testing & Total \\
\hline Ten & 369 & 66 & 435 \\
\hline Twenty & 371 & 66 & 437 \\
\hline Fifty & 379 & 68 & 447 \\
\hline Hundred & 351 & 62 & 413 \\
\hline Five hundred & 341 & 61 & 402 \\
\hline One thousand & 371 & 66 & 437 \\
\hline
\end{tabular}

Fig 1: Distribution of Indian Currency Denominations

The method we proposed is transfer learning, in which there are different models for image classification. A framework has been defined for a better understanding of transfer learning, which is defined as follows:-

We can use many transfer learning models like EfficientNet, Xception, VGG16, MobileNet, and many others. We have trained and tested our dataset through several models with different hyperparameters, as shown in fig below.

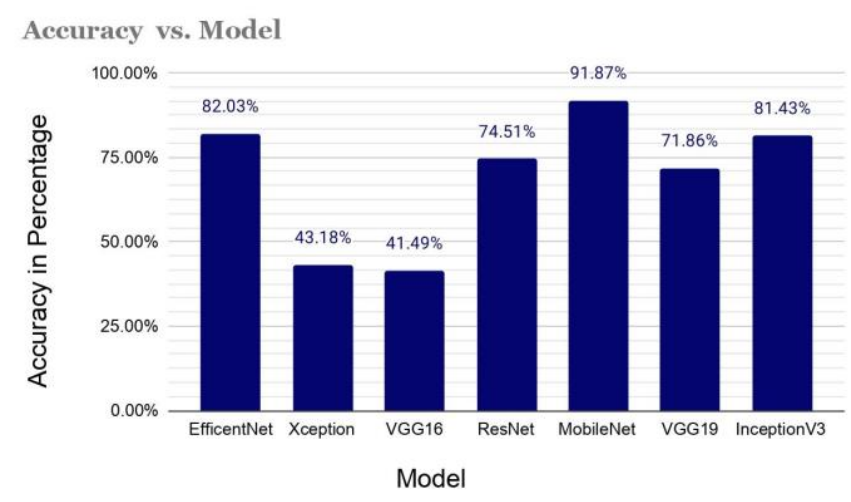

After training 6-7 models, we got the best results for MobileNet, EfficentNet, InceptionV3. We can conclude from the bar graph that the Mobilenet has the highest accuracy of classifying as an image correctly and confidently, so for further training and testing of models, we used MobleNet as our model for classifying the images. MobileNets are based on a streamlined architecture that utilizes depth-wise detachable convolutions to construct lightweight and compact deep neural networks. We enlist two simple global hyper-parameters that efficiently trade-off between latency and accuracy. These hyper-parameters enable the model builder to select the rightsized model for their application created on the problem's limitations. We shall be using Mobilenet as it is compact in its architecture. It uses depth wise separable convolutions, which implies it conducts a single convolution on each color channel 


\section{International Journal of Engineering Applied Sciences and Technology, 2020 \\ Vol. 5, Issue 6, ISSN No. 2455-2143, Pages 335-340 \\ Published Online October 2020 in IJEAST (http://www.ijeast.com)}

rather than blending all three and flattening it. For MobileNets, the depthwise convolution pertains to a single filter to each input channel. The pointwise convolution then applies a $1 \times 1$ convolution to incorporate the outputs of the depthwise convolution. A standard convolution, both filters and combines inputs into a new set of outputs in one step. The depthwise separable convolution slices this into two layers, a separate layer for filtering and a separate layer for combining. This factorization has the consequence of drastically reducing computation and model size.

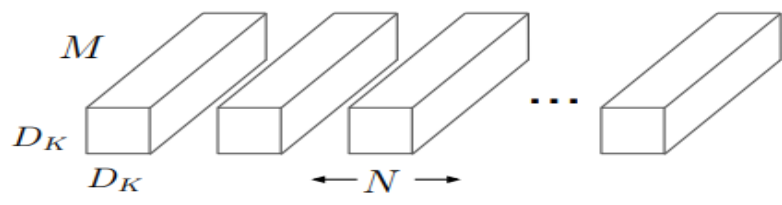

(a) Standard Convolution Filters

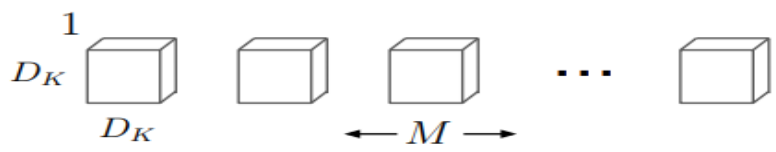

(b) Depthwise Convolutional Filters

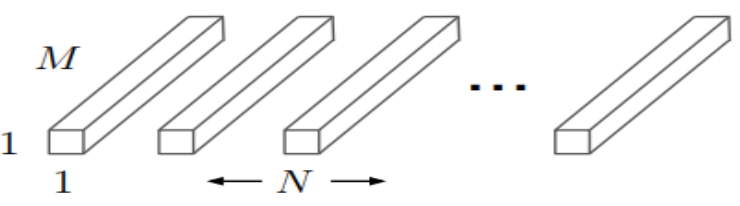

(c) $1 \times 1$ Convolutional Filters called Pointwise Convolution in the context of Depthwise Separable Convolution

The widespread architecture of the Mobilenet is as follows, having 30 layers with

1. convolutional layer with stride 2

2. depthwise layer

3. pointwise layer that doubles the number of channels

4. depthwise layer with stride 2

5. pointwise layer that doubles the number of channels

MobileNet is a neural network architecture that operates extremely efficiently on mobile devices. Its architecture glimpses like as shown in the figure below:

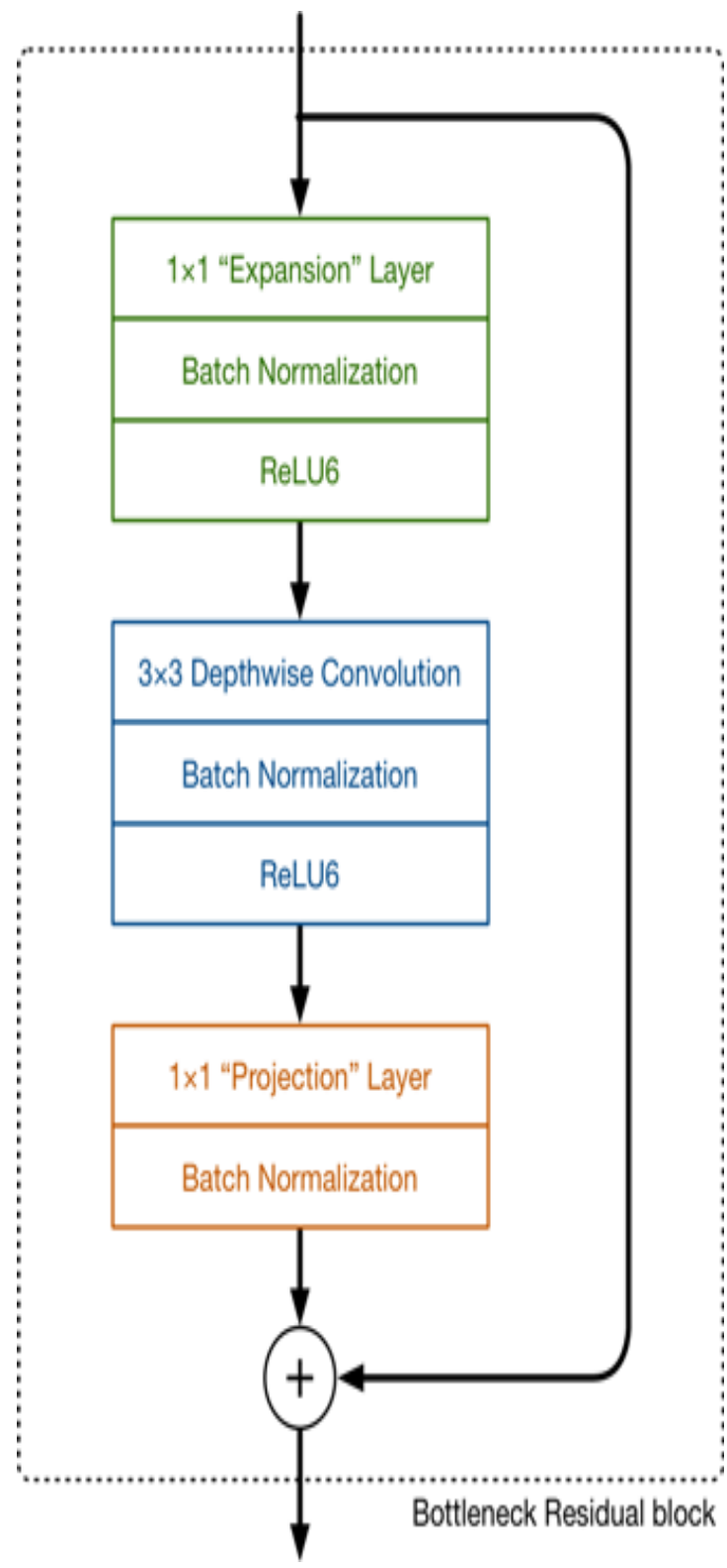

It requires inadequate maintenance, thus accomplishing incredibly competently, delivering high speed. There are several categories of pre-trained models with the network size in memory and disk being proportional to the number of parameters being used. The network's speed and power consumption are proportional to the number of MACs (Multiply-Accumulates), a measure of the number of fused Multiplication and Addition operations 


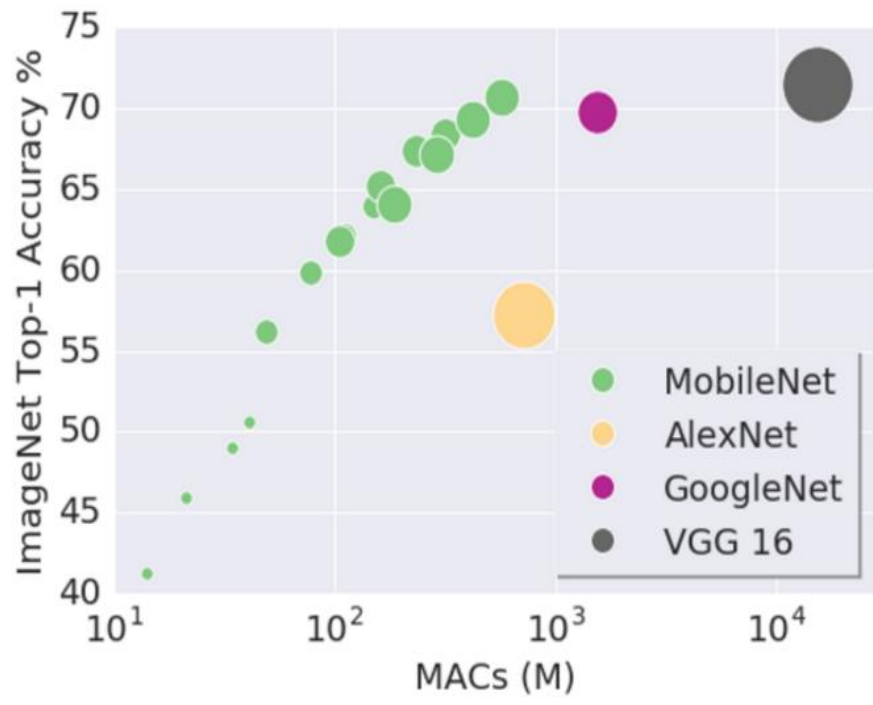

IV. EXPERIMENTAL RESULTS

The figure below is the representation of the accuracy in percentage for different numbers of epochs. We can conclude that from different batch sizes, we got the highest percentage of accuracy in batch size 16 .
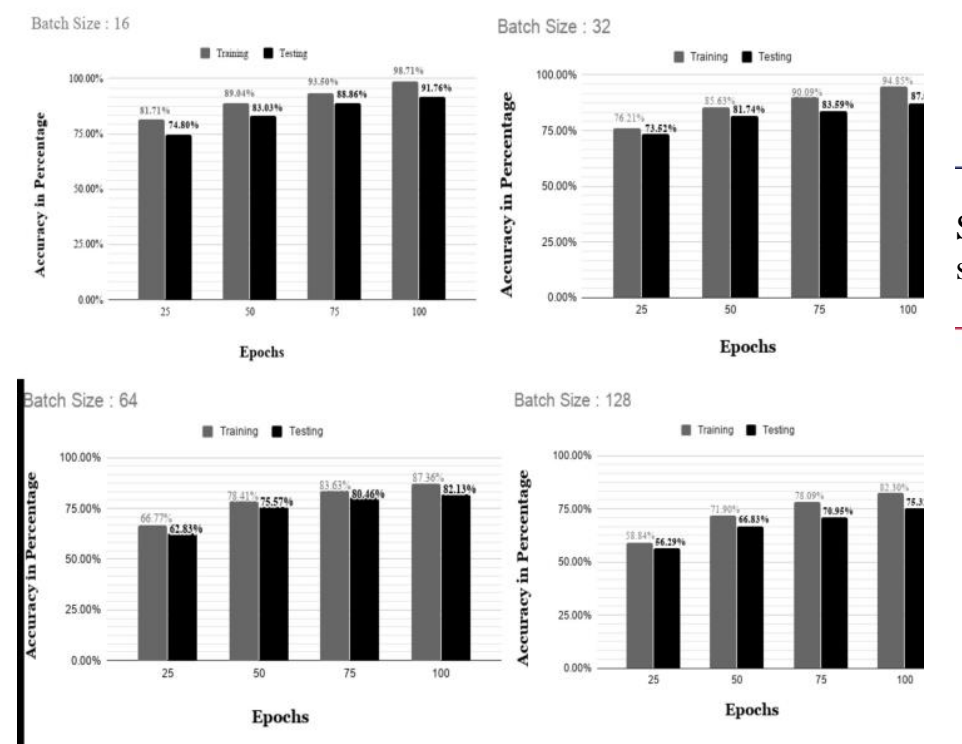

The web application UI is shown below with the working of model and output.

The following steps for recognition of currency notes:

Step 1: Browse the picture you want to recognize which type of currency your picture is and upload it.

\section{Indian Currency Classifier}

Classifies 6 types of Indian Currency 500,1000

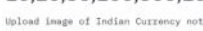

Step 2: When you have uploaded the picture, it shows some output as processing/classifying the image.

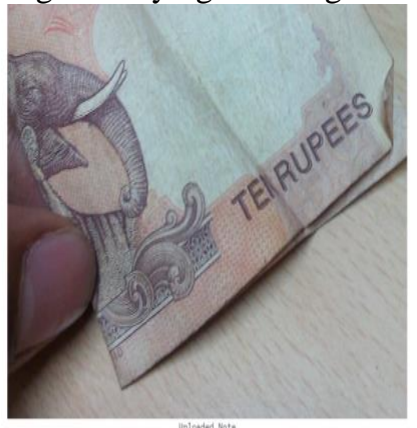

Step 3: After the model has classified the image correctly, it shows the output, as shown in the picture below.
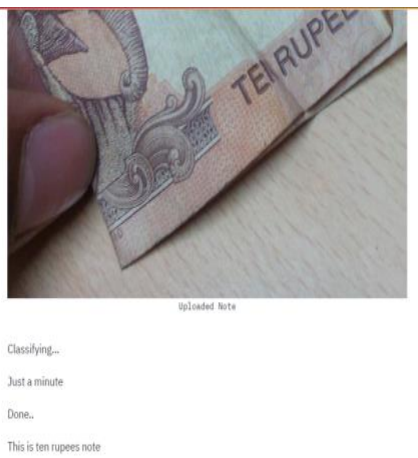

The deployment of the android application is shown below in 3 steps, same as a web application:

Step 1:Click to upload the photo from gallery. 
International Journal of Engineering Applied Sciences and Technology, 2020

Vol. 5, Issue 6, ISSN No. 2455-2143, Pages 335-340

Published Online October 2020 in IJEAST (http://www.ijeast.com)

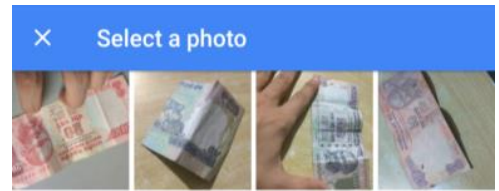

Click On

Gallery icon

to select an

image

4

$\bullet$

-

Step 2:Allow to access the photos.

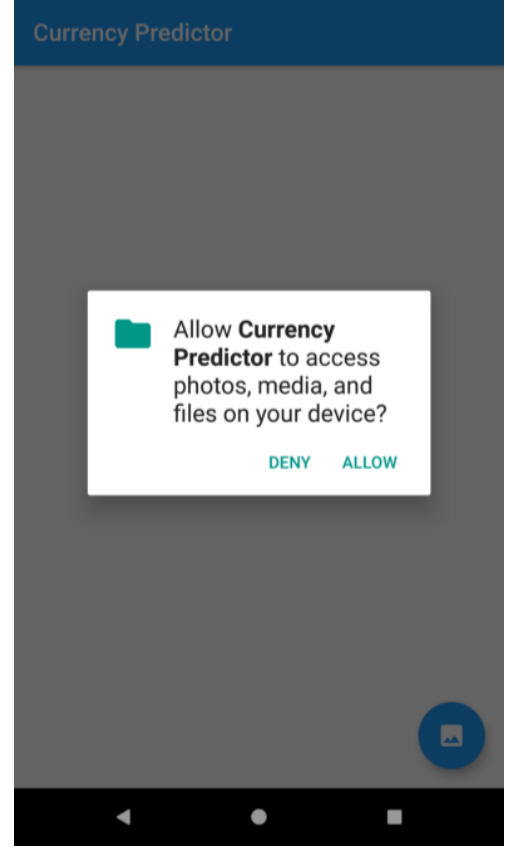

Step 3:Browse and select the photo.

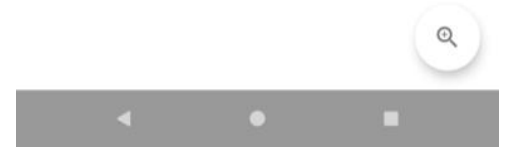

Step 4: The final output of the model

Currency Predictor

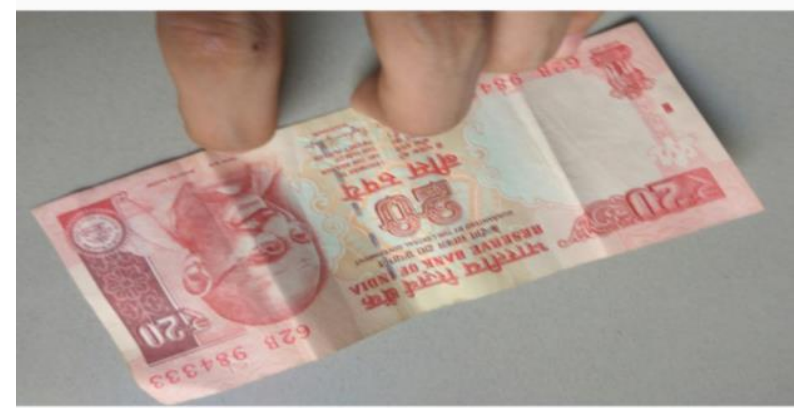

This is twenty rupees.

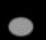

口 


\section{International Journal of Engineering Applied Sciences and Technology, 2020 \\ Vol. 5, Issue 6, ISSN No. 2455-2143, Pages 335-340 \\ Published Online October 2020 in IJEAST (http://www.ijeast.com)}

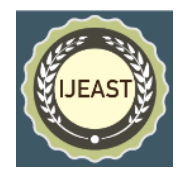

\section{CONCLUSION}

In this paper, we have explained the model we constructed to classify Indian currency notes. We have also elucidated the notion behind this, i.e., convolution neural network (CNN) algorithm and transfer learning; which helped extract features of the currency note. We furthermore experimented for each model to intend for sufficient accuracy. We tried on models like VGG16, ResNet, MobileNet, and supplementary models using some hyper-parameters like an epoch, batch size, and learning rate. Out of all the models we tested, MobileNet bestowed us with the highest accuracy for our dataset. We then deployed the model with a web-app using a library called Streamlit in python and an android app using framework Flutter developed by google to make it serviceable for the user.

Our current restrictions include a lack of risk analysis. Due to given time constraints, we aim to optimize and better accuracy in the next phase. In the future, we will improve on a better dataset, which in turn provides hereafter giving much better accuracy and results. We also aim at the web-app uploaded on the cloud to make it public since currently, ours works on local machines. We can also aim to work on the UI part of the webapp and an android app to make it more understandable for the end-user in the future.

\section{REFERENCES}

[1] R. Vaillant, C. Monrocq, and Y. Le Cun.(1994) "An Original approach for the localization of objects in images". IEE Proceedings on Vision, Image, and Signal Processing, 141(4).

[2] Henry A. Rowley, Shumeet Baluja, and Takeo Kanade.(1995). "Human face detection in visual scenes". CMCS-95-158R, Carnegie Mellon University,Also available at http://www.cs.cmu.edu/ har/faces.html.

[3] Frosini, A. Gori, M. and Priami, P.(1996), "A neural network-based model for paper currency recognition and verification", In Journal of Neural Networks, IEEE Transactions on Vol 7, No 6,pp 1482-1490,

[4] Takeda, F. and Nishikage, T.(2000). "Multiple kinds of paper currency recognition using neural network and application for Euro currency", In Proceedings of the IEEE-INNS-ENNS International Joint Conference on Neural Networks, IJCNN 2000, Vol. 2, pp 143-147.

[5] Er-Hu Zhang Bo Jiang Duan Jing-hong and Zheng-Zhong Bian.(2003)."Research on paper currency recognition by neural networks", In Proceedings of International Conference on Machine Learning and Cybernetics, Vol. 4, pp 2193-2197.

[6] A. Vila and N. Ferrer and J. Mantecón and D. Bretón and J.F.García.(2006).Development of a fast and nondestructive procedure for characterizing and distinguishing original and fake euro notes,In Journal of Analytica Chimica Acta, Vol. 559, No. 2, pp. 257-263.
[7] Hassanpour, H. and Yaseri, A. and Ardeshiri, G.(2007). "Feature extraction for paper currency recognition", In Proceedings of 9th International Symposium on Signal Processing and its Applications, ISSPA, pp. 1-4, February..

[8] Girshick et al.(2014): "Rich feature hierarchies for accurate object detection and semantic segmentation,IEEE Conference on Computer Vision and Pattern Recognition, pp. $580-587$.

[9] Ryutaro Kitagawa, Yoshihiko Mochizuki, Satoshi Iizuka , Edgar Simo-Serra , Hiroshi Matsuki , Naotake Nator, Hiroshi Ishikawa.(2017).15th IAPR International Conference on Machine Vision Applications (MVA) Nagoya University, Nagoya, Japan.

[10] P. Gidveer, S.R. Darade, Automatic Recognition of Fake Indian Currency Note, International Conference on Electrical Power and Energy Systems (ICEPES), Bhopal, India, pp. 290-294

[11] He, K., Zhang, X., Ren, S., \& Sun, J. (2016). Deep residual learning for image recognition. In Proceedings of the IEEE conference on computer vision and pattern recognition (pp. 770-778).

[12] Simonyan, K., \& Zisserman, A. (2014). Very deep convolutional networks for large-scale image recognition. arXiv preprint arXiv:1409.1556. 